\title{
DETERMINAÇÃO DA TAXA DE AMOSTRAGEM E ANALISE DO EFEITO DO FALSEAMENTO DOS COMPONENTES DE FREQUENCIA DE UM SINAL DE IDENTIFICAÇÃO
}

\author{
T. S. S. DANTAS, R.R. FONSECA e F. V. da SILVA
}

Universidade Estadual de Campinas, Faculdade de Engenharia Química, Laboratório de Controle e Automação de Processos - LCAP

\begin{abstract}
RESUMO - A determinação da taxa de amostragem, em controle digital, é uma etapa importante no projeto de um sinal para identificação de sistemas dinâmicos a partir de dados do processo. É uma etapa que freqüentemente é dada pouca atenção pelos Engenheiros Químicos na área de controle de processos. Este trabalho tem como objetivo aplicar um método de determinação de taxa de amostragem que satisfaz o teorema de Shannon-Nyquist e esclarecer como o falseamento de frequências (aliasing) é potencialmente prejudicial a identificação de sistemas. Um estudo de caso é realizado através de simulação a fim de demonstrar a importância deste trabalho.
\end{abstract}

\section{INTRODUÇÃO}

O procedimento de amostragem de dados produzidos pelo sistema é uma característica inerente do controle digital. É inevitável que a amostragem cause a perda de alguma informação, porém é possível minimizar este efeito.Esta perda de informação ocorrida na amostragem é melhor descrita no domínio da frequência. Suponha que um sinal $S(t)$ é amostrado com um período de amostragem $\mathrm{T}$, tal que $S(t)=S(k T)$, para $\mathrm{k}=(1,2, \ldots)$. Seja $\omega_{S}=2 \pi / T$ a frequência de amostragem e $\omega_{N}=\omega_{S} / 2$ a frequência de Nyquist. Já é conhecido que uma função senoidal com frequência maior que $\omega_{N}$ não pode, quando amostrada, ser distinguida de uma frequência no intervalo $\left[-\omega_{N}, \omega_{N}\right]$ (Ogata, 1997). Por consequência, se esta frequência de amostragem for tal que $|\omega|>\omega_{N}$, haverá sobreposição de sinais tal que $\cos \omega k T=\cos \bar{\omega} k T$ e $\sin \omega k T=\sin \bar{\omega} k T$, sendo $-\omega_{N}>\bar{\omega}>\omega_{N}$, indicando falseamento do sinal amostrado.

O fenômeno de falseamento de frequências, ou aliasing, ocorre porque partes do espectro de um sinal amostrado que correspondem às frequências mais altas que $\omega_{N}$, serão interpretadas como contribuições de frequências mais baixas que $\omega_{N}$ (Equações 1-5). Consequentemente o espectro do sinal amostrado será uma superposição de diferentes partes do espectro original.

$$
\varphi_{T}(s)=\varphi_{c}\left(\omega+k \omega_{s}\right)
$$


Onde $\varphi_{T}$ é espectro total, $\varphi_{c}$ é o espectro em tempo contínuo, $\omega$ é a frequência do sinal contínuo, $\omega_{S}$ a frequência de amostragem do sinal, $\mathrm{k}$ uma constante de relação entre as frequências de amostragem e de Nyquist. $\operatorname{Com} R_{c}(\tau)$ sendo a função de auto-correlação para o espectro em tempo contínuo e $R_{T}(l T)$ a para o espectro total, as funções de $\varphi_{c}$ e $\varphi_{T}$ são obtidas pela transformada de Fourier de suas respectivas funções de auto-correlação (Aguirre, 1997).

$$
\begin{gathered}
R_{c}(\tau)=\lim _{L \rightarrow \infty} \frac{1}{L} \int_{0}^{L} E s(t) s(t+\tau) d t \\
\varphi_{c}(\omega)=\int_{-\infty}^{\infty} R_{c}(\tau) e^{-i \omega \tau} d \tau \\
R_{T}(l T)=\lim _{N->\infty} \frac{1}{N} \sum_{k=1}^{N} E s(k T) s(k T+l T) \\
\varphi_{T}(\omega)=T \sum_{l=-\infty}^{\infty} R_{T}(l T) e^{-i \omega l T}
\end{gathered}
$$

A determinação da taxa de amostragem é, portanto, um aspecto importante no desenvolvimento de sinais para identificação de sistemas, uma vez que é uma técnica de modelagem para controle de processos na qual modelos dinâmicos são construídos através de dados observados do sistema (Ljung, 1987). Se o sistema estiver sendo amostrado com uma taxa de amostragem não adequada, os algoritmos de detecção de estrutura e estimação de parâmetros, mesmo os mais avançados, não produzirão modelos que representem o comportamento do sistema adequadamente.

Segundo o teorema de Shannon-Nyquist, reapublicado por Shannon (1998), a frequência de amostragem $\omega_{S}$ deve ser pelo menos duas vezes maior do que a frequência mais alta $\omega_{N}$ presente no sinal analógico, em que $\omega_{N}$ é a frequência de Nyquist, representada pela Equação 6.

$$
\omega_{S} \geq 2 * \omega_{N}
$$

\section{ESTUDO DE CASO}

A influência de diferentes taxas de amostragem em um sistema digital foi avaliada em um modelo de dois reatores isotérmicos denominados 1 e 2, em série, não interativos, com reação de primeira ordem e operando com vazão de entrada e saída constantes a volume constante. $\mathrm{O}$ reator 1 é alimentado com uma corrente de reagente com concentração $C a_{i n} \mathrm{e}$ descarregado no reator 2 com uma corrente de concentração $C a_{1}$. O modelo fenomenológico dos reatores em série podem ser representados pelas equações 7 e 8 , respectivamente.

$$
\begin{aligned}
& \frac{d C a_{1}}{d t}=\frac{F}{V_{1}} C a_{i n}-\frac{F}{V_{1}} C a_{1}-k_{0} C a_{1} \\
& \frac{d C a_{2}}{d t}=\frac{F}{V_{2}} C a_{1}-\frac{F}{V_{2}} C a_{2}-k_{0} C a_{2}
\end{aligned}
$$

Utilizando transformada de Laplace e transformando em variável desvio obteve-se um modelo de função de transferência de segunda ordem $G_{P}(s)$ representada pela Equação 9, relacionando a saída do segundo reator com a entrada do primeiro reator. 
Tabela 1 - Parâmetros do modelo dos reatores CSTR em série

\begin{tabular}{cc} 
Parâmetros do modelo & Valor(Unidade) \\
\hline $\boldsymbol{F}$ (Vazão) & $6.5 \mathrm{~L} / \mathrm{min}$ \\
$\boldsymbol{V}_{\mathbf{1}, \mathbf{2}}$ (Volume) & $2.4 \mathrm{~L}$ \\
$\boldsymbol{k}_{\mathbf{0}}$ (constante cinética) & $0.017 \mathrm{~min}^{-1}$ \\
\hline
\end{tabular}

$$
G_{P}(s)=\frac{C_{2}(s)}{C a_{\text {in }}(s)}=\frac{0.9987}{0.1346 s^{2}+0.734 s+1}
$$

Pela Figura 1, determina-se a largura de banda do processo e a frequência de Nyquist através do diagrama de Bode que corresponde aoponto de $-3 \mathrm{~dB}$, que representa o valor absoluto da magnitude para a qual a função de transferência de potência assume metade do seu valor máximo, indicando a largura de banda (Woyczynski, 2011). A largura de banda do processo foi determinada como $1.74 \mathrm{rad} / \mathrm{s}$, sendo esta justamente a frequência máxima de interesse do sistema. Forçou-se então uma variação senoidal com frequência de $1.74 \mathrm{rad} / \mathrm{s}$ na concentração $C a_{i n}$ na corrente de alimentação do reator 1 e verificou-se a variação nos valores de concentração $C a_{2}$ na corrente de saída do reator 2 de forma contínua, como mostrado na Figura 2. Os valores de $C a[\mathrm{~mol} / \mathrm{L}]$ estão apresentados em variável desvio em torno de um determinado regime permanente arbitrário, tal que $C_{A}^{\prime}=C_{A}(t)-C_{A, R P}$.

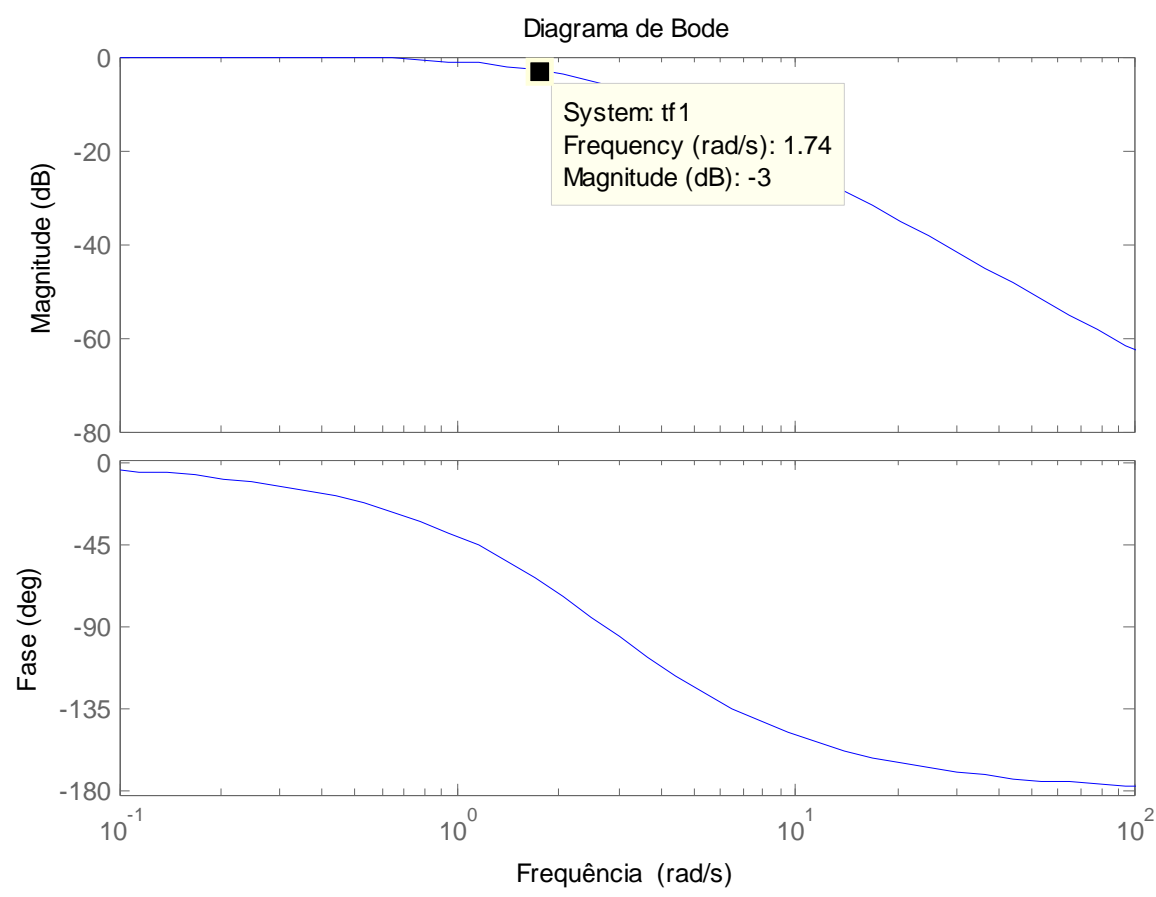

Figura1 - Diagrama de Bode para a função de transferência $G_{p}(s)$. 


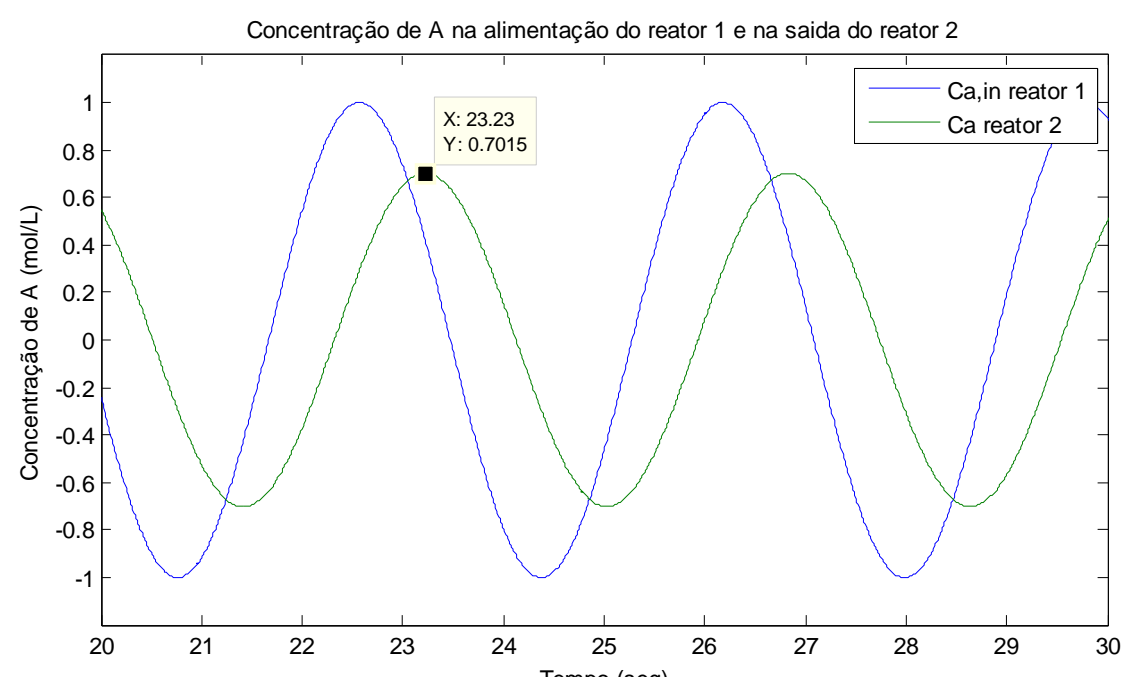

Figura 2 - Concentrações $C a_{i n}$ e $C a_{2}$ a uma frequência de $1,74 \mathrm{rad} / \mathrm{s}$.

Vale mencionar que pelo diagrama de bode, na frequência de $1.74 \mathrm{rad} / \mathrm{s}$ há um deslocamento no ângulo de fase de 65.2 graus entre o sinal continuo de saída $\mathrm{Ca}_{2}$ em relação à entrada $C a_{i n}$, devido principalmente ao atraso de transporte de massa em decorrência ao tempo de residência do reagente nos reatores. A atenuação entre as amplitudes dos valores de $\mathrm{Ca}_{i n} \mathrm{e} C \mathrm{a}_{2}$ é aproximadamente em variável desvio igual a 0.707 [mol/L] na escala absoluta, que na escala logarítmica equivale a $-3 \mathrm{~dB}$.

Ao realizar a amostragem do sinal de $C a_{2}$ a uma taxa de 1.74 [rad/s], observa-se pela Figura 3 que o período de amostragem esta em sincronia com a oscilação do sinal contínuo de $C a_{2}$, ou seja, cada amostragem captura o mesmo valor de concentração, proporcionando uma falsa interpretação de que a concentração na saída do reator 2 é constante. Isto corrobora com os resultados apresentados no espectro de potência na Figura 4, que será discutido mais detalhadamente adiante. A taxa de amostragem correta de $\mathrm{Ca}_{2}$, seguindo o teorema de Shannon-Nyquist, tem que ser pelo menos $3.48 \mathrm{rad} / \mathrm{s}$.

É possível observar qualitativamente o fenômeno de falseamento na Figura 3 quando a concentração $\mathrm{Ca}_{2}$ é amostrada a $1.64[\mathrm{rad} / \mathrm{s}] \mathrm{e}$ a $1.84[\mathrm{rad} / \mathrm{s}]$. Ambas frequências estão à \pm 0.1 [rad/s] da frequência de Nyquist, apesar disso observa-se que, aparentemente, as funções senoidais discretas estão ambas com a mesma frequência de oscilação, mesma amplitude porém com um atraso de fase de alguns graus, cerca de 5,7 graus pelo diagrama de Bode da Figura 1,estando ambos gráficos na mesma escala de tempo. O mesmo pode ser observado qualitativamente para os pares de frequência $1.74 \pm 0.2[\mathrm{rad} / \mathrm{s}]$ e $1.74 \pm 0.3[\mathrm{rad} / \mathrm{s}]$, também apresentados na Figura 3.

Uma análise mais quantitativa em relação à frequência de sinais amostrados ou séries temporais pode ser realizada através do espectro de potência apresentado na Figura 4. Através da transformada rápida de Fourier(FFT) um sinal arbitrário é decomposto em um somatório de senos e cossenos com seus respectivos coeficientes. Porém é mais interessante conhecer o conteúdo absoluto de informação que está presente em uma determinada frequência, independentemente se faz parte da função seno ou cosseno. Esta informação está contida no valor absoluto dos coeficientes da FFT para cada frequência. Para obter a potência do sinal, 
eleva-se o quadrado o valor absoluto do coeficiente da série de Fourier e isto é considerado a potência do sinal [W/Hz], conforme Storey (2002).

Sinal da concentração $\mathrm{Ca}$ amostrado com diferentes taxas de amostragem

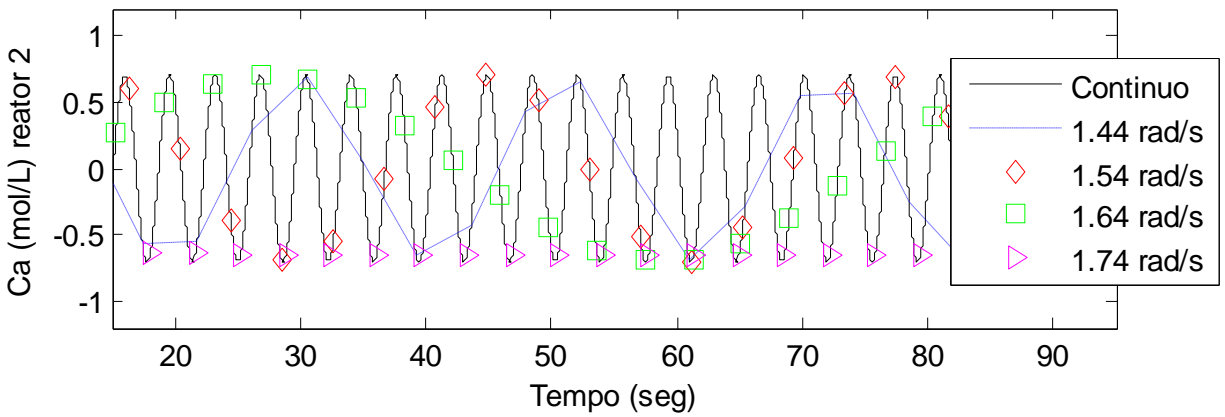

Sinal da concentração $\mathrm{Ca}$ amostrado com diferentes taxas de amostragem

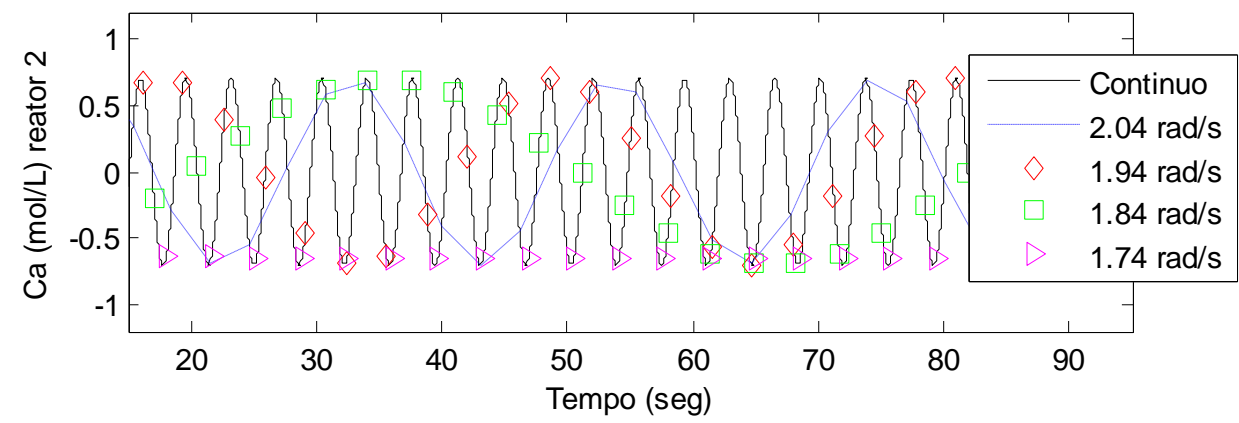

Figura 3 - Sinal da concentração de $\mathrm{Ca}(\mathrm{mol} / \mathrm{L})$ no reator 2

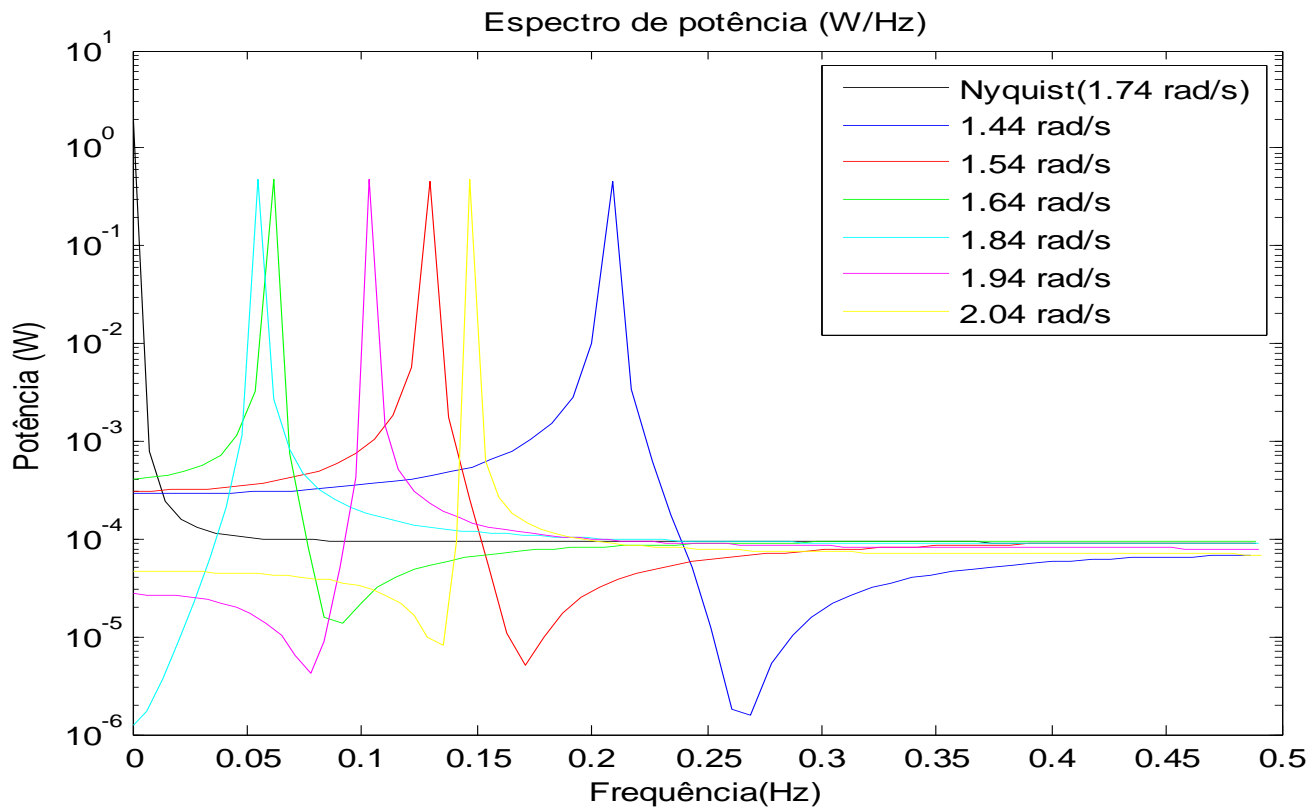

Figura 4 - Espectro de potência 
A frequência dominante do sinal em um espectro de potência é determinada pelo maior valor absoluto do coeficiente da FTT elevado ao quadrado, ou seja, são os picos do espectro que foi gerado em uma taxa de amostragem constante. Estes picos indicam a frequência dominante do sinal com aquela taxa de amostragem. Por exemplo, na frequência de amostragem de $1.74 \mathrm{rad} / \mathrm{s}$, a frequência dominante no espectro de potência é $0 \mathrm{~Hz}$ como se observa na Figura 4, ou seja, não há oscilação nos pontos amostrados.

Quando a frequência de amostragem de uma função senoidal vai aumentando até o limite superior da largura de banda (frequência de Nyquist), a frequência dominante do sinal resultante vai diminuindo como foi discutido anteriormente e pode ser visto qualitativamente na Figura 3. Este efeito ocorre quando $\omega_{\text {Nyquist }}$ é ultrapassada, pois cada incremento na taxa de amostragem causa um aumento na frequência dominante do sinal amostrado.

Porém como pode ser visto na Figura 4, o sinal da concentração $C a_{2}$ amostrado com taxas de $1.64[\mathrm{rad} / \mathrm{s}]$ e $1.84[\mathrm{rad} / \mathrm{s}]$ possuem frequências dominantes quase iguais. Foi dito anteriormente que na Figura 3, a amostragem a $1.64[\mathrm{rad} / \mathrm{s}]$ e $1.84[\mathrm{rad} / \mathrm{s}]$ apresentavam comportamento similar no domínio do tempo qualitativamente, aparentando na verdade ser a mesma onda senoidal, só que com maior ângulo de fase. O que é um fato interessante, pois $1.84[\mathrm{rad} / \mathrm{s}]$ é maior que $1.64[\mathrm{rad} / \mathrm{s}]$. Isto é a característica que define o falseamento de frequências, quando uma taxa de amostragem superior a largura de banda do processo se apresenta como uma frequência menor que a frequência de Nyquist. A mesma análise é válida para as taxas de amostragem de $\omega_{\text {Nyquist }} \pm 0.2[\mathrm{rad} / \mathrm{s}]$ e $\omega_{\text {Nyquist }} \pm 0.3[\mathrm{rad} / \mathrm{s}]$.

Apesar do espectro descrever apenas alguns características de um sinal, muitas propriedades relacionadas a identificação de processos dependem apenas do espectro dos sinais envolvidos (Ljung, 1987). No projeto de sinais em identificação de sistemas, é importante que o sinal de identificação excite todas as frequências relevantes do processo, diz então que o sinal de identificação é persistentemente excitante. Um sinal de identificação branco possui potência espectral não-nula em todas as frequências, como observado na Figura $5 b$, e função de autocorrelação nula exceto na origem (Aguirre, 1997).

Os processos químicos possuem em geral um perfil passa-baixa, similar a um filtro de ruídos passa baixa, onde o perfil dominante de potência do sinal de saída se encontra em frequências mais baixas. O que condiz com observações reais, uma vez que os processos químicos possuem dinâmicas mais lentas. Em regime permanente, ao se realizar uma mudança no estado de referência (setpoint) em um reator CSTR ou em uma coluna de destilação, a dinâmica do processo para se atingir um novo estado estacionário ocorre em um tempo razoável, podendo durar horas ou dias. Enquanto que em sistemas elétricos as constantes de tempo são pequenas, favorecendo o deslocamento das frequências dominantes do processo para valores mais altos. Um sinal de identificação com características que favorecem processos mais lentos pode ser visto na Figura 6. 

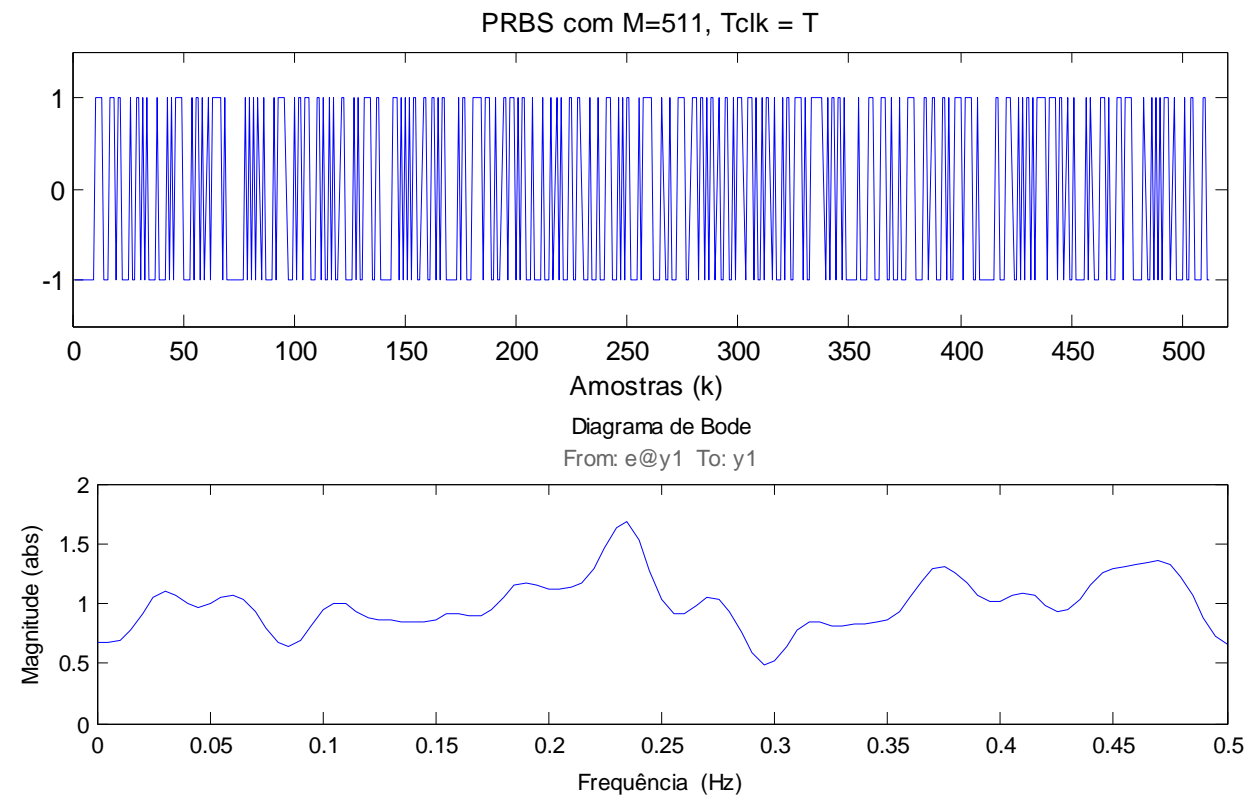

Figura 5 - Sinal de identificação PRBS branco.
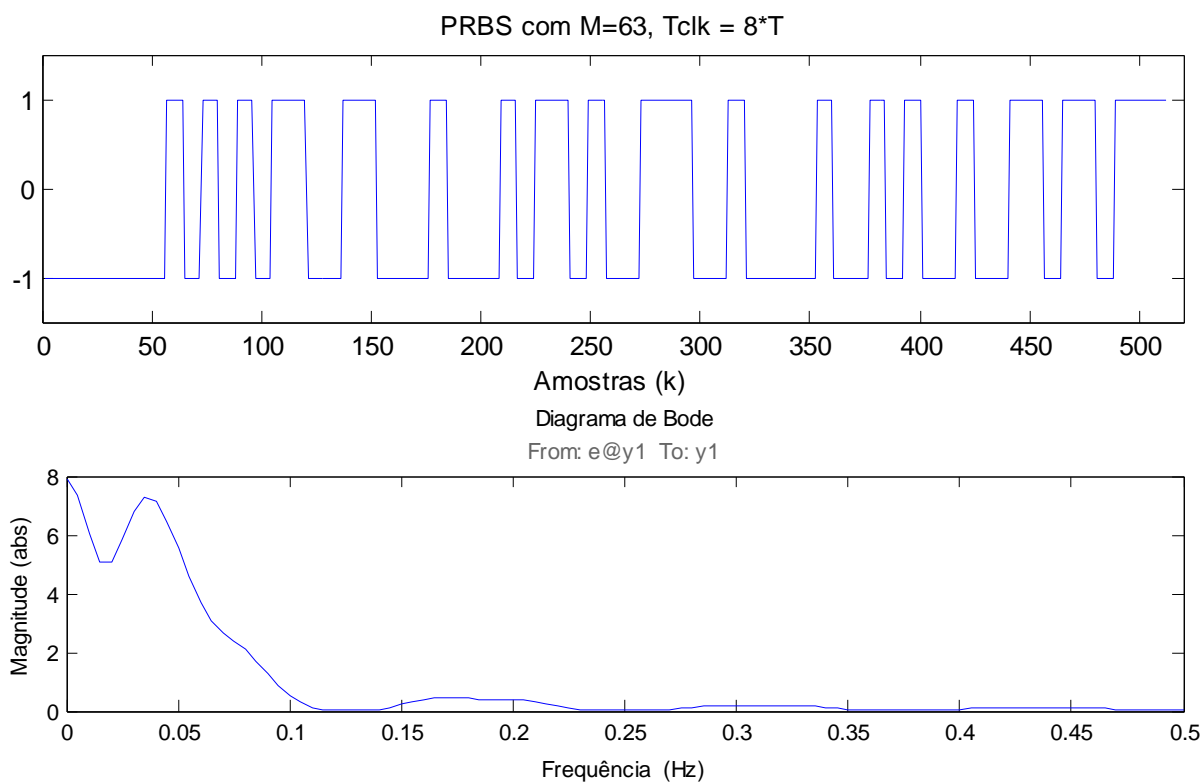

Figura 6 - Sinal de identificação PRBS "passa-baixa"

\section{CONCLUSÃO}

O falseamento de frequência pode potencialmente interferir na identificação de sistemas. Porque componentes de alta frequência dobram e deslocam-se para regiões do espectro onde tipicamente encontra-se informação relevante à dinâmica dos processos químicos e ao comportamento estático do modelo "real" do processo. Podendo interferir e/ou amplificar coeficientes de modelos causando polarização de parâmetros ou até mesmo alterando a estrutura e ordem do modelo. 


\section{REFERÊNCIAS}

AGUIRRE, L.A. Introdução a identificação de sistemas. Editora UFMG. 2007.

LJUNG, L. System identification: Theory for the User. Prentice Hall. 1987.

OGATA, K. Engenharia de Controle Moderno, Prentice Hall, 1997.

SHANNON, C. Communication in the presence of noise. Proceedings of IEEE. Vol. 86, No. 2, 1998.

STOREY, B. D. Computing Fourier Series With MATLAB. Notas de aula. 2002.

WOYCZYNSKI, W.A. A First course in Statistics for Signal Analysis. 2:aedição. Springer. 2011. 